\title{
Segurança e educação durante a COVID-19: prevalência, fatores associados e planos de reabertura da Faculdade de Enfermagem
}

\author{
Safety and education during COVID-19: prevalence, associated factors and reopening plans of the
} School of Nursing

\section{Seguridad y educación en COVID-19: prevalencia, factores asociados, planes de reapertura en una}

Ricardo de Mattos Russo Rafael ${ }^{1}$ (1)

Luiza Mara Correia ${ }^{1}$ (1)

Alex Simões de Mello ${ }^{1}$ (1)

Juliana Amaral Prata ${ }^{1}$ (C)

Cristiane Helena Gallasch ${ }^{1}$ (D)

Eugenio Fuentes Pérez Junior ${ }^{1}$ (b)

Frances Valeria Costa e Silva ${ }^{1}$ (D)

Lucia Helena Garcia Penna ${ }^{1}$ (D)

Jaime Caravaca Morera²

Karen Lucas Breda ${ }^{3}$ (C)

Helena Maria Scherlowski Leal David ${ }^{1}$ (1)

1. Universidade do Estado do Rio de Janeiro,

Faculdade de Enfermagem. Rio de Janeiro,

RJ, Brasil.

2. Universidad de Costa Rica, Escuela de

Enfermería. San José, Costa Rica.

3. University of Hartford, College of Education, Nursing and Health Professions. West

Hartford, Connecticut, USA.
Autor correspondente:

Ricardo de Mattos Russo Rafael

E-mail: prof.ricardomattos@gmail.com.

Recebido em 12/12/2020.

Aprovado em 17/03/2021.

DOl:https://doi.org/10.1590/2177-9465-EAN-2020-0528

\section{Resumo}

Objetivo: analisar a prevalência e os fatores associados à triagem da COVID-19 e propor as estratégias necessárias para a reabertura das Escolas de Enfermagem universitárias para as aulas presenciais. Métodos: um estudo transversal foi projetado a partir da linha de base de uma pesquisa longitudinal baseada na Internet de uma Escola Brasileira de Enfermagem. Com o resultado ordinal (classificação operacional COVID-19), a análise de dados foi conduzida usando um modelo de regressão multinomial. Resultados: um total de 498 participantes foi incluído na análise e revelou $67,27 \%$ de casos suspeitos e 11,65\% de casos confirmados de COVID-19. O uso de máscaras, o transporte público e fazer parte da linha de frente dos trabalhadores da saúde foram estatisticamente associados a um histórico positivo para a COVID-19. Conclusão: os planos de reabertura de Escolas de Enfermagem devem integrar o uso de equipamentos de proteção pessoal, o transporte público e a triagem da COVID-19 na educação.

Palavras-chave: Universidades; Segurança; Enfermagem; Infecção por Coronavírus; Pandemias.

\section{Abstract}

Objective: to analyze the prevalence and factors associated with COVID-19 screening and propose the necessary strategies for the reopening of university Nursing Schools to face-to-face classes. Methods: aa cross-sectional study was designed from the baseline of a longitudinal Internet-based survey of a Brazilian School of Nursing. With the ordinal outcome (COVID-19 operationa classification), data analysis was conducted using a multinomial regression model Results: a total of 498 participants were included in the analysis and revealed $67.27 \%$ suspected and $11.65 \%$ confirmed cases of COVID-19. Wearing masks, public transportation, and being part of frontline healthcare workers were statistically associated with a positive history for COVID-19. Conclusion: plans to reopen Nursing Schools must integrate the use of personal protective equipment, public transportation, and COVID-19 screening into education.

Keywords: Universities; Safety; Nursing; Coronavirus infection; Pandemics.

\section{Resumen}

Objetivo: analizar la prevalencia y los factores asociados al rastreo de COVID-19 y proponer las estrategias necesarias para la reapertura de Escuelas Universitarias de Enfermería para clases presenciales. Métodos: se diseñó un estudio transversal a partir de la línea de base de una investigación longitudinal basada en Internet de una Escuela Brasileña de Enfermería. Con el resultado ordinal (clasificación operativa COVID-19), se realizó el análisis de datos mediante un modelo de regresión multinomial Resultados: se incluyeron en el análisis un total de 498 participantes que revelaron el $67,27 \%$ de los casos sospechosos y el $11,65 \%$ de los casos confirmados de COVID-19. El uso de máscaras, el transporte público y ser parte de la primera línea de los trabajadores de la salud se asociaron estadísticamente con un historial positivo de COVID-19. Conclusión: los planes de reapertura de Escuelas de Enfermería deben integrar el uso de equipos de protección personal, transporte público y el rastreo de COVID-19 en la educación.

Palabras clave: Universidades; Seguridad; Enfermería; Infección por coronavirus; Pandemias. 


\section{INTRODUÇÃO}

Com a declaração da nova pandemia de Coronavírus (COVID-19) pela Organização Mundial da Saúde (OMS), um conjunto de ações destinadas a reduzir a velocidade da progressão da curva epidemiológica foi iniciado pela comunidade científica e por organizações internacionais. ${ }^{1,2}$ Portanto, a distância física era o principal método para conter a pandemia na época. ${ }^{3,4}$

Devido ao alto risco de contágio entre as pessoas, a pandemia rapidamente levou ao fechamento de locais públicos e instituições educacionais em todo o mundo. ${ }^{5} \mathrm{Na}$ tentativa de minimizar o impacto das perdas associadas à interrupção das atividades educacionais nas universidades, foi desenvolvido o uso de estratégias de ensino/aprendizagem mediadas por plataformas digitais, constituindo, assim, um desafio pedagógico contínuo tanto para o corpo docente universitário quanto para os estudantes. Essa realidade exige uma avaliação de risco das possibilidades de retorno às aulas presenciais e às atividades clínicas com estudantes de Enfermagem de graduação.

A discussão sobre a suscetibilidade de crianças e jovens adultos na cadeia de transmissão da COVID-19 acentuou a necessidade da tomada de decisões relativas à reabertura de escolas e universidades e na decisão individual de adotar ou não as estratégias de saúde para evitar o contágio. ${ }^{6-10}$ Além da ciência, a negação e a desinformação como política ocorreram em alguns países, incluindo o Brasil. ${ }^{11,12}$ Além disso, houve falhas importantes do governo federal brasileiro no rastreamento da doença e na prestação de cuidados coordenados aos pacientes. ${ }^{3,13}$ Apesar de o Brasil ser um dos países com um alto número de casos e mortes pela COVID-19 e uma capacidade fraca de aplanar a curva epidemiológica da doença, muita discussão e conflito ocorreram relacionados com o retorno às aulas presenciais.

Além disso, não é conhecida a taxa de soroprevalência entre professores, funcionários e estudantes nas universidades brasileiras, especialmente nos cursos de profissionais de saúde, tais como as Escolas de Enfermagem. Os estudos sobre este tema podem acrescentar conhecimentos cruciais para ajudar a moldar as políticas públicas. O objetivo deste trabalho é analisar a prevalência e os fatores associados à triagem da COVID-19 e propor as estratégias necessárias para a reabertura de Escolas de Enfermagem baseadas em universidades para as aulas presenciais e os laboratórios clínicos.

\section{MÉTODOS}

Este estudo transversal baseia-se na linha de base da pesquisa "COVID-19 Pandemia e vida acadêmica: coorte sobre a situação da doença, condições sociais e experiências acadêmicas", e 0 artigo seguiu as diretrizes recomendadas. ${ }^{14,15} \mathrm{~A}$ pesquisa foi realizada em uma Escola de Enfermagem de nível universitário localizada no Rio de Janeiro, um programa que ocupa um lugar importante entre os programas de graduação e pós-graduação em Enfermagem no Brasil.

Uma pesquisa baseada na Internet foi projetada para este estudo com um subconjunto analisando o período de 4 de junho a 3 de setembro de 2020. A população-alvo era a comunidade acadêmica da Escola de Enfermagem, incluindo os professores, o pessoal administrativo e os estudantes. Uma amostra de conveniência foi utilizada para todos os professores, pessoal administrativo e estudantes que tiveram um relacionamento formal com a Escola de Enfermagem durante o período de referência. Participantes potenciais do estudo foram 326 estudantes de graduação, 410 estudantes completando cursos de especialização, 88 estudantes de pós-graduação (nível de mestrado e doutorado), 104 professores de Enfermagem e 26 funcionários administrativos, totalizando 954 participantes potenciais.

O recrutamento ocorreu em duas fases e teve como alvo professores, pessoal administrativo e estudantes oficialmente ligados à Escola de Enfermagem. Na primeira fase de coleta de dados, uma carta de apresentação explicando o estudo, um link para o consentimento informado e os instrumentos de coleta de dados foram enviados aos participantes potenciais por meio de seu e-mail institucional. Em casos de não resposta e após três dias, um novo e-mail foi enviado. Posteriormente, a equipe de pesquisa fez contato telefônico para convidar os potenciais participantes que, após duas tentativas, não completaram a pesquisa baseada na Internet. Tentou-se fazer contato telefônico adicional a cada três dias em horários diferentes durante o dia e à noite, incluindo os dias de semana e fins de semana. Ao aceitar a participação, o link para o instrumento on-line foi novamente encaminhado por e-mail, constituindo a segunda fase da coleta de dados. Se os participantes potenciais não preenchessem a pesquisa, um novo e-mail era enviado. Após cinco tentativas de contato, com três e-mails e duas tentativas de contato telefônico, os potenciais participantes que não responderam foram excluídos do estudo. Um total de 498 participantes respondeu ao instrumento e constituiu a amostra final da pesquisa.

Para evitar múltiplas entradas pelo mesmo participante e considerando a impossibilidade de bloqueio de IP devido ao compartilhamento de computadores por muitos estudantes, o instrumento de coleta de dados continha informações (nome completo, endereço de e-mail e outros dados) que possibilitavam a dupla checagem com os bancos de dados institucionais. A fim de garantir a integridade das informações, o banco de dados foi armazenado criptografado e sem informações pessoais. Um backup diário foi realizado para evitar a perda de dados e para remover o armazenamento da rede da Internet.

A definição de caso de COVID-19 utilizada neste estudo é a classificação operacional de casos adotada pelo Ministério da Saúde do Brasil. ${ }^{16}$ Os participantes que relataram um histórico de testes ou de confirmação de critérios clínico-epidemiológicos foram considerados casos confirmados.

Os critérios de confirmação dos testes foram: um teste de Biologia Molecular (RT-PCR em tempo real) com um resultado detectável (positivo) para SARS-CoV-2 em uma amostra clínica coletada entre $04^{\circ}$ e o $10^{\circ}$ dias ou um Teste Imunológico (sorologia clássica ou teste rápido) com resultados positivos de $\operatorname{lgM}$ ou IgG em amostras coletadas após o sétimo dia. Em casos sem 
confirmação laboratorial, os critérios clínico-epidemiológicos foram: relato de contato domiciliar ou próximo com uma pessoa com um teste positivo para o SARS-CoV-2 nos sete dias anteriores ao início dos sintomas.

Uma definição de caso suspeito foi adotada para os indivíduos que fizeram testes inconclusivos ou não realizaram os testes de Biologia Molecular, Sorologia Clássica ou Teste Rápido e apresentaram sintomas compatíveis com a síndrome respiratória (síndrome da gripe e Síndrome Respiratória Aguda Grave) sem histórico de contato com uma pessoa confirmada para o SARS-CoV-2 nos sete dias que antecederam os sintomas.

As variáveis de resultado foram compostas pelo conjunto de manifestações clínicas respiratórias, o teste diagnóstico para a COVID-19 e o histórico positivo de contato confirmado e, consequentemente, os respectivos intervalos de tempo, conforme indicado na definição operacional do caso pelo Ministério da Saúde brasileiro. As covariáveis investigadas neste estudo foram compostas de características sociodemográficas, clínicas e de saúde. Um grupo de risco positivo foi considerado quando o participante relatou o histórico positivo em uma ou mais destas características: idade superior a 60 anos; gravidez; pós-parto; obesidade e condições crônicas (doença cardíaca, diabetes, doença hepática, neuropatia, imunodeficiência, nefropatia, câncer e doenças respiratórias crônicas).

A prevalência e os respectivos intervalos de confiança de 95\% foram calculados para as definições de casos da COVID-19 (descartados, suspeitos e confirmados) e para a classificação de casos confirmados tais como os confirmados por exame laboratorial e os confirmados por critérios clínico-epidemiológicos.

As análises bivariadas foram realizadas com base na estimativa da Odds Ratio (ORb) bruta, usando o qui-quadrado como teste de hipótese. Todas as variáveis com valor $p<0,20$ foram incluídas no modelo de regressão inicial, aplicando a regressão multinomial como uma técnica para estimar os fatores associados ao resultado. A partir do modelo inicial, foi aplicada uma técnica gradual para trás, removendo individualmente as covariáveis não significativas até chegar aos modelos finais (com todas as variáveis com um valor $p<0,05$ ). Para a avaliação global do ajuste do modelo, foi utilizado o Teste de Wald. A análise e o processamento do banco de dados foram realizados utilizando o software Stata SE $15 . .^{17}$

A pesquisa foi conduzida de acordo com os princípios éticos envolvendo os seres humanos em conformidade com a legislação internacional e brasileira e aprovada pelo Comitê de Ética em Pesquisa do IRB (Conselho de Revisão Institucional) da universidade (Número de aprovação: 4.058.958). Todos os participantes concordaram em participar, assinando a autorização eletrônica de Consentimento Livre e Esclarecido.

\section{RESULTADOS}

No total, 498 dos 954 participantes potenciais completaram os questionários eletrônicos, resultando em uma taxa de resposta de 52,20\%. Ao analisar o subgrupo populacional, foram observadas as seguintes taxas de resposta: 76,38\% $(n=249 / 326)$ para os estudantes de graduação; $28,53 \%(n=117 / 410)$ para os estudantes de especialização; $36,26 \%(n=32 / 88)$ para os estudantes de mestrado e doutorado; $80,76(n=84 / 104)$ para os professores e $61,53 \%(n=16 / 26)$ para o pessoal administrativo.

A amostra foi composta predominantemente por mulheres $(\mathrm{n}=444 ; 89,16 \%)$, brancas $(\mathrm{n}=251 ; 50,50 \%)$, com idade média de 30,15 anos (DP 11,53), residentes na capital do Estado do Rio de Janeiro ( $\mathrm{n}=352 ; 70,68 \%)$, com renda individual de até US $\$ 544,47$ por mês ( $n=304 ; 62,42 \%$ ) e vivendo (em média) com 0,77 (DP 0,65) pessoas por cômodo na casa. As características clínicas da amostra indicam que 41,97\% (n=209) dos participantes estão no grupo de risco para a COVID-19 e que $63,86 \%(n=318)$ deles vivem com um membro da família considerado de risco. Além disso, 57,63\% $(n=287)$ da amostra tem seguro de saúde privado em cima do seguro de saúde público.

Especificamente, sobre as medidas de saúde e a exposição durante a pandemia da COVID-19, 67,87\% ( $n=338)$ dos participantes trabalhavam em casa, $42,97 \%(n=214)$ receberam ou visitaram familiares e amigos, $72,29 \%(n=360)$ usaram o transporte público para chegar à universidade e outros ambientes de trabalho e 96,79\% ( $n=482)$ relataram sempre usar máscaras. Além disso, 29,92\% $(n=149)$ dos participantes eram ativos como trabalhadores de saúde clínica no atendimento direto aos pacientes, em média, 1,04 (DP 1,72) dias por semana.

A Figura 1 mostra os casos da COVID-19 de acordo com a classificação operacional. Observa-se que 67,27\% $(n=335$; IC 95\%: 63,01 - 71,26) da amostra foi classificada como casos suspeitos, 21,08\% ( $n=105$; IC 95\%: 17,71 - 24,90) como casos negativos e 11,65\% ( $n=58$; IC 95\%: 9,10 - 14,78) como casos confirmados da COVID-19. Com base em uma classificação mais específica, é possível extrair dois subconjuntos de casos confirmados: 7,63\% ( $n=38 / 498$; IC 95\%: 5,59 - 10,32) com confirmação de teste laboratorial e 4,02\% (n=20/498; IC 95\%: 2,60 - 6,15) confirmados por critérios clínico-epidemiológicos.

Com base nestes resultados, chama-se a atenção para a proporção de pessoas testadas para a COVID-19: 24,79\% ( $n=123 ;$ IC 95\%:21,10-28,69). Dos participantes que relataram síndrome respiratória ( $\mathrm{n}=395 ; 79,31$; IC 95\%: 75,52 - 82,66), apenas 17,97\% ( $n=71 / 395$; IC 95\%: 14,48 - 22,09) relataram um histórico de exames para a COVID-19. Dos participantes com Síndrome Respiratória Aguda Grave ( $n=27 / 498$; 5,42\%; IC 95\%: 3,74 - 7,80), nove (33,33\%) relataram testes laboratoriais para a COVID-19. Vale notar que 23 participantes $(4,62 \%$; IC 95\%; 3,08 - 6,86) relataram a tomografia de tórax. Destes, três relataram imagens compatíveis com pneumonia viral e apresentaram um teste laboratorial positivo para a COVID-19, sendo omitidos do fluxograma na Figura 1.

Tabelas 1 e 2 apresentam a prevalência da classificação dos casos e os fatores sociodemográficos, clínicos e sanitários associados para confirmar e descartar os casos da COVID-19. A maior prevalência de casos suspeitos e, consequentemente, o mais alto ORb estão associados às variáveis raça/etnia, sexo, menores níveis de treinamento acadêmico e menor renda, além de estarem associados ao trabalho de casa da variável 


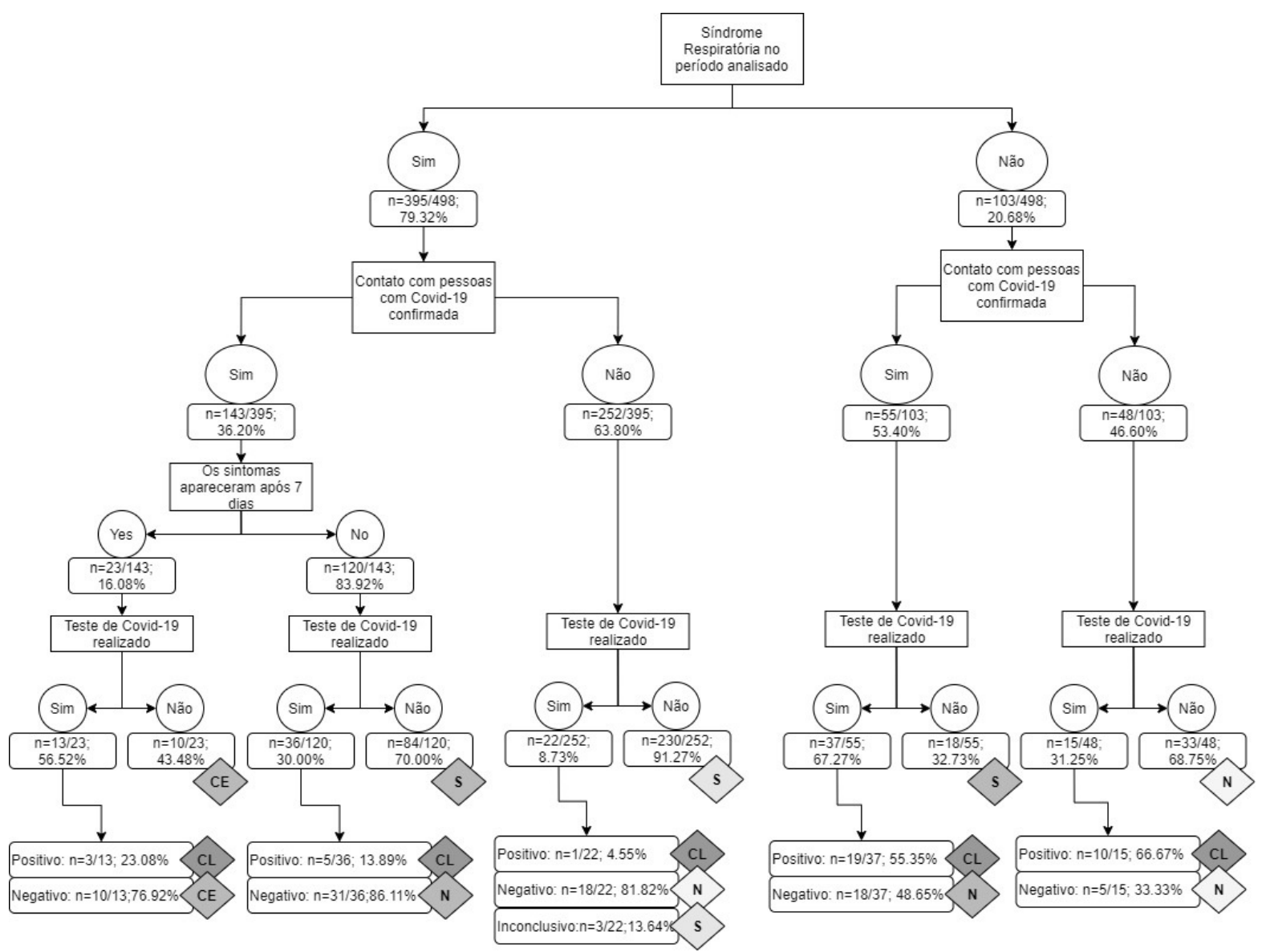

Figura 1. Casos de Covid-19 de acordo com a classificação operacional do estudo e de acordo com as manifestações clínicas, o contato domiciliar com os casos confirmados, o desempenho e o resultado dos testes diagnósticos.

Legenda: CL: Confirmação laboratorial; CE: Confirmação por critérios clínico-epidemiológicos; S: Caso suspeito; N: Caso negativo (descartado).

$(p<0,001)$, trabalhadores da linha de frente da saúde com atendimento direto ao paciente $(<0,001)$ e dias da semana no atendimento direto ao paciente $(<0,001)$ e uso de máscaras $(p$ $0,004)$. A menor prevalência de casos foi encontrada entre os estudantes de graduação $(4,02 \%)$, enquanto os estudantes que concluíram os programas de especialização de Enfermagem tiveram a maior prevalência da COVID-19 (25,64\%).

Finalmente, a Tabela 3 mostra o modelo final de regressão multinomial. $O$ uso do transporte público reduziu o efeito sobre os casos descartados (ORa 0,54; p 0,016). Isto significa que, ao utilizar casos negativos como referência, a ORa é 1,80 (CI95\% 1,10 - 2,93; p 0,019). Observa-se, considerando que a amostra mostrou o esmagador uso predominante de máscaras, que só foi possível calcular o efeito da variável na relação casos suspeitos/casos descartados, apresentando ORa 5,19 ( $p$ 0,008). Finalmente, a participação variável no trabalho de atendimento direto na linha de frente da saúde mostrou um efeito estatisticamente significativo para as duas categorias de resposta: caso suspeito/caso descartado $(2,91 ; p<0,001)$ e caso suspeito/caso confirmado $(7,83 ; p<0,001)$. Nenhuma interação foi identificada a partir deste modelo.

\section{DISCUSSÃO}

Este estudo é o primeiro a apresentar os dados da triagem da COVID-19 em uma Escola Brasileira de Enfermagem, mostrando que a prevalência de casos confirmados (histórico de teste positivo e contato domiciliar com o caso de COVID-19) foi de cerca de $11 \%$. Estes resultados são compatíveis com os de outros estudos internacionais cuja soroprevalência da SARS-CoV-2 foi entre um e 10\%.18-22 No entanto, os dados deste estudo diferem dos de outros estudos baseados na população brasileira.

No Rio de Janeiro, entre 14 e 27 de abril, foram realizadas pesquisas, encontrando uma prevalência bruta de 4,0\% (IC 95\% 3,3 - 4,7) de anti-SARS-CoV-2 em 2.857 doadores de sangue. As estimativas ajustadas resultaram em uma prevalência de $3,3 \%$ (IC 95\% 2,6 - 4,1) após a aplicação dos pesos para a população do Estado do Rio de Janeiro e após o ajuste para a sensibilidade e a especificidade dos testes..$^{23}$ Estimativas semelhantes foram encontradas em um estudo transversal em série realizado em 11 municípios do vizinho Estado brasileiro do Espírito Santo, mostrando a prevalência de 2,1\% (IC 95\% 1,67 - 2,52) em 6.393 pessoas testadas. ${ }^{24}$ Duas pesquisas de soroprevalência domésticas e consecutivas realizadas em 133 
Tabela 1. Prevalência e Odds Ratio bruto de casos suspeitos versus os casos da Covid-19 confirmados e descartados por características sociodemográficas

\begin{tabular}{|c|c|c|c|c|c|c|c|}
\hline \multirow{2}{*}{ Variável } & \multirow{2}{*}{$\begin{array}{c}\text { Caso } \\
\text { suspeito }\end{array}$} & \multicolumn{3}{|c|}{ Caso descartado } & \multicolumn{3}{|c|}{ Caso confirmado } \\
\hline & & n (\%) & ORb (IC 95\%)* & $\mathbf{P}$ & $\mathrm{n}(\%)$ & ORb (IC 95\%)* & $\mathbf{P}$ \\
\hline $\begin{array}{l}\text { Idade: Média } \\
\text { (DP) }\end{array}$ & $29,63(12,04)$ & $30,34(10,70)$ & $1,00(, 98-1,02)$ & 0,572 & $32,81(9,65)$ & $1,02(, 99-1,04)$ & 0,054 \\
\hline Raça/etnia & & - & - & - & - & - & - \\
\hline Negra & $168(69,71)$ & $44(18,26)$ & 1,00 & - & $29(12,03)$ & 1,00 & - \\
\hline Branca & $165(65,74)$ & $60(23,90)$ & $1,39(0,89-2,16)$ & 0,148 & $25(10,36)$ & $0,91(0,51-1,62)$ & 0,754 \\
\hline $\begin{array}{l}\text { Asiática/ } \\
\text { Indígena }\end{array}$ & $1(20,00)$ & $1(20,00)$ & $3,82(0,23-62,26)$ & 0,347 & $3(60,00)$ & $17,38(1,74-172,86)$ & 0,015 \\
\hline \multicolumn{8}{|l|}{ Gênero } \\
\hline Mulher & $304(68,47)$ & $87(19,59)$ & 1,00 & - & $53(11,94)$ & 1,00 & - \\
\hline Homem & $31(57,41)$ & $18(33,33)$ & $2,03(1,08-3,80)$ & 0,027 & $5(9,26)$ & $0,92(0,34-2,49)$ & 0,877 \\
\hline \multicolumn{8}{|l|}{$\begin{array}{l}\text { Categoria } \\
\text { acadêmica }\end{array}$} \\
\hline $\begin{array}{l}\text { Estudantes de } \\
\text { graduação }\end{array}$ & $194(77,91)$ & $45(18,07)$ & 1,00 & - & $10(4,02)$ & 1,00 & - \\
\hline $\begin{array}{l}\text { Estudantes de } \\
\text { especialização }\end{array}$ & $56(47,86)$ & $31(26,50)$ & $2,39(1,38-4,11)$ & 0,002 & $30(25,64)$ & $10,39(4,79-22,56)$ & $<0,001$ \\
\hline $\begin{array}{l}\text { Mestres } \\
\text { doutorandos }\end{array}$ & $16(50,00)$ & $10(31,25)$ & $2,69(1,15-6,32)$ & 0,023 & $6(18,75)$ & $7,27(2,34-22,59)$ & 0,001 \\
\hline Professores & $56(66,67)$ & $18(21,43)$ & $1,38(0,74-2,58)$ & 0,304 & $10(11,90)$ & $3,46(1,37-8,74)$ & 0,009 \\
\hline Técnicos & $13(81,25)$ & $1(6,25)$ & $0,33(0,04-2,60)$ & 0,294 & $2(12,50)$ & $2,98(0,59-15,06)$ & 0,186 \\
\hline \multicolumn{8}{|c|}{ Área residencial } \\
\hline Capital & $101(69,18)$ & $30(20,55)$ & 1,00 & - & $15(10,27)$ & 1,00 & - \\
\hline $\begin{array}{l}\text { Outros } \\
\text { municípios }\end{array}$ & $234(66,48)$ & $75(21,31)$ & $1,07(0,66-1,75)$ & 0,758 & $43(12,22)$ & $1,24(0,66-2,33)$ & 0,509 \\
\hline \multicolumn{8}{|l|}{$\begin{array}{l}\text { Renda (em } \\
\text { dolar)** }\end{array}$} \\
\hline Até \$181,49 & $169(78,24)$ & $37(17,13)$ & 1,00 & - & $10(4,63)$ & 1,00 & - \\
\hline $\begin{array}{l}\$ 181,50 a \\
\$ 544,47\end{array}$ & $47(53,41)$ & $25(28,41)$ & $2,43(1,33-4,43)$ & 0,004 & $16(18,18)$ & $5,75(2,45-13,51)$ & $<0,001$ \\
\hline $\begin{array}{l}\$ 544,48 a \\
\$ 807,46\end{array}$ & $48(64,00)$ & $13(17,33)$ & $1,24(0,61-2,51)$ & 0,556 & $14(18,67)$ & $4,93(2,05-11,79)$ & $<0,001$ \\
\hline $\begin{array}{l}\$ 807,46 \text { a } \\
\$ 1.814,91\end{array}$ & $43(58,11)$ & $17(22,97)$ & $1,80(0,93-3,51)$ & 0,081 & $14(18,92)$ & $5,50(2,29-13,24)$ & $<0,001$ \\
\hline $\begin{array}{l}\text { Mais do que } \\
\$ 1.814,91\end{array}$ & $19(55,88)$ & $11(32,35)$ & $2,64(1,16-6,02)$ & 0,021 & $4(11,76)$ & $3,56(1,01-12,45)$ & 0,047 \\
\hline
\end{tabular}

* Odds Ratio bruto (ORb) foi calculado utilizando como referência os casos suspeitos. ** A conversão de valores foi realizada em 24 de setembro de 2020 e utilizou a cotação de $\$ 1,00$ para $R \$ 0,18$.

cidades sentinela em todos os Estados brasileiros resultaram em $1,39 \%$ de casos positivos (347/24.995) e 2,40\% (746/31.128) em ambas as investigações, respectivamente..$^{25}$ Este estudo estimou 7,5\% (IC 95\%: 4,2 e 12,2\%) dos casos da COVID-19 para o Rio de Janeiro e apresentou resultados mais próximos das conclusões gerais encontradas.

Algumas situações podem ajudar a entender essas diferenças de prevalência. A primeira situação é a característica 
Tabela 2. Prevalência e Odds Ratio bruto de casos suspeitos versus os casos da Covid-19 confirmados e descartados por características clínicas e sanitárias.

\begin{tabular}{|c|c|c|c|c|c|c|c|}
\hline \multirow{2}{*}{ Variável } & \multirow{2}{*}{$\begin{array}{c}\text { Caso } \\
\text { suspeito } \\
\mathrm{n}(\%) \text { ou } \\
\text { média (DP) }\end{array}$} & \multicolumn{3}{|c|}{ Caso descartado } & \multicolumn{3}{|c|}{ Caso confirmado } \\
\hline & & $\begin{array}{l}\mathrm{n}(\%) \text { ou } \\
\text { média (DP) }\end{array}$ & ORb (IC 95\%)* & $\mathbf{P}$ & $\begin{array}{c}\mathrm{n}(\%) \text { ou } \\
\text { média (DP) }\end{array}$ & ORb (IC 95\%)* & $\mathbf{P}$ \\
\hline \multicolumn{8}{|l|}{ Grupo de risco } \\
\hline Não & $196(67,82)$ & $59(20,42)$ & 1,00 & - & $34(11,76)$ & 1,00 & - \\
\hline Sim & $139(66,51)$ & $46(22,01)$ & $1,10(0,71-1,71)$ & 0,675 & $24(11,48)$ & $0,99(0,56-1,75)$ & 0,987 \\
\hline \multicolumn{8}{|c|}{ Membro da família do grupo de risco } \\
\hline Não & $118(65,56)$ & $40(22,22)$ & 1,00 & - & $22(12,22)$ & 1,00 & - \\
\hline Sim & $217(68,24)$ & $65(20,44)$ & $0,88(0,56-1,39)$ & 0,593 & $36(11,32)$ & $0,89(0,50-1,58)$ & 0,691 \\
\hline \multicolumn{8}{|l|}{ Seguro de saúde } \\
\hline Não & $145(68,72)$ & $42(19,91)$ & 1,00 & - & $24(11,37)$ & 1,00 & - \\
\hline Sim & $190(66,20)$ & $63(21,95)$ & $1,14(0,73-1,79)$ & 0,553 & $34(11,85)$ & $1,08(0,61-1,90)$ & 0,787 \\
\hline \multicolumn{8}{|c|}{ Visitou a família ou os amigos } \\
\hline Não & $188(66,20)$ & $62(21,83)$ & 1,00 & - & $34(11,97)$ & 1,00 & - \\
\hline Sim & $147(68,69)$ & $43(20,09)$ & $0,89(0,57-1,38)$ & 0,597 & $24(11,21)$ & $0,90(0,51-1,59)$ & 0,723 \\
\hline \multicolumn{8}{|l|}{ Trabalho em casa } \\
\hline $\begin{array}{l}\text { Sim ou } \\
\text { desempregado }\end{array}$ & $264(78,11)$ & $58(17,16)$ & 1,00 & - & $16(4,73)$ & 1,00 & - \\
\hline $\begin{array}{l}\text { Não; intervalo de } \\
\text { trabalho reduzida }\end{array}$ & $32(41,56)$ & $23(29,87)$ & $3,27(1,78-6,00)$ & $<0,001$ & $22(28,57)$ & $11,34(5,41-23,80)$ & $<0,001$ \\
\hline $\begin{array}{l}\text { Não; intervalo de } \\
\text { trabalho normal }\end{array}$ & $39(46,99)$ & $24(28,92)$ & $2,80(1,56-5,01)$ & 0,001 & $20(24,10)$ & $8,46(4,04-17,71)$ & $<0,001$ \\
\hline \multicolumn{8}{|c|}{ Linha de frente do setor de saúde } \\
\hline Não & $269(77,08)$ & $61(17,48)$ & 1,00 & - & $19(5,44)$ & 1,00 & - \\
\hline Sim & $66(44,30)$ & $44(29,53)$ & $2,94(1,83-4,71)$ & $<0,001$ & $39(26,17)$ & $8,37(4,54-15,41)$ & $<0,001$ \\
\hline $\begin{array}{l}\text { Dias da semana } \\
\text { na linha de frente } \\
\text { da saúde: média } \\
\text { (DP) }\end{array}$ & $0,67(1,48)$ & $1,50(1,88)$ & $1,34(1,18-1,51)$ & $<0,001$ & $2,34(1,88)$ & $1,64(1,41-1,91)$ & $<0,001$ \\
\hline $\begin{array}{l}\text { Pessoa por sala: } \\
\text { Média (DP) }\end{array}$ & $0,77(0,65)$ & $0,74(0,36)$ & $0,90(0,59-1,37)$ & 0,635 & $0,78(0,96)$ & $0,99(0,66-1,50)$ & 1,000 \\
\hline \multicolumn{8}{|c|}{ Uso de transporte público (ônibus, trem, barco, etc.) } \\
\hline Não & $80(57,97)$ & $38(27,54)$ & 1,00 & - & $20(14,49)$ & 1,00 & - \\
\hline $\operatorname{Sim}$ & $255(70,83)$ & $67(18,61)$ & $0,55(0,34-0,89)$ & 0,014 & $38(10,56)$ & $0,60(0,33-1,08)$ & 0,089 \\
\hline \multicolumn{8}{|l|}{ Uso de máscara } \\
\hline Sempre & $328(68,05)$ & $96(19,92)$ & 1,00 & - & $58(12,03)$ & 1,00 & - \\
\hline $\begin{array}{l}\text { Na maioria do } \\
\text { tempo }\end{array}$ & $7(43,75)$ & $9(56,25)$ & $4,39(1,59-12,11)$ & 0,004 & - & - & - \\
\hline
\end{tabular}

* Odds Ratio bruto (ORb) foi calculado utilizando como referência os casos suspeitos.

heterogênea da distribuição da doença, sendo afetada pelas características da população e da organização dos sistemas de saúde. ${ }^{25} \mathrm{~A}$ segunda situação pode ser a multiplicidade de testes diagnósticos usados nos estudos, que naturalmente produzem medidas diferentes, dependendo da precisão dos testes. Entretanto, acredita-se que a principal situação a ser considerada é que os resultados deste estudo mostram medidas de grupos com diferentes exposições. 
Tabela 3. Modelo final de regressão multinomial*.

\begin{tabular}{lcccccc}
\hline \multirow{2}{*}{ Variável } & \multicolumn{2}{c}{ Caso descartado } & & & \multicolumn{2}{c}{ Caso confirmado } \\
\cline { 2 - 3 } & ORa (IC 95\%)** & $\mathbf{p}$ & & ORa (IC 95\%)** & $\mathbf{p}$ \\
\hline Usa transporte público: não/sim & $0,54(0,33-, 89)$ & $\mathbf{0 , 0 1 6}$ & & $0,63(0,33-1,19)$ & 0,155 \\
Uso de máscara: sempre/na maioria do tempo & $5,19(1,54-17,38)$ & $\mathbf{0 , 0 0 8}$ & & - & - \\
Linha de frente do setor de saúde: não/sim & $2,91(1,77-4,80)$ & $<0,001$ & & $7,83(4,24-14,46)$ & $<0,001$ \\
\hline
\end{tabular}

* Odds Ratio Ajustada (ORa) foi calculada usando como referência os casos suspeitos. ** Estatística do Teste de Wald: 58,81 ( $<<0,001)$.

Notavelmente, aproximam-se dos resultados deste estudo os estudantes de graduação $(4,02 \%)$ com prevalência encontrada em pesquisas nacionais e internacionais baseadas na população. ${ }^{22-26}$ Os alunos têm estado sem aulas no local e mantendo o distanciamento social e outras práticas de saúde pública desde o início da pandemia da COVID-19. ${ }^{23-25}$ O mesmo vale para o pessoal administrativo, que não foi exposto aos riscos das unidades de saúde. Assim, acredita-se que os riscos de contágio destes dois grupos são semelhantes aos riscos da população em geral.

O efeito do uso de máscara sobre a maior prevalência de casos confirmados não deve ser interpretado à luz da causalidade e do risco de infecção. Acontece que o uso deste equipamento de proteção, como é conhecido para reduzir o risco de infecção, também reduz a probabilidade de ocorrência de casos suspeitos. Assim, além dos ganhos óbvios na prevenção da infecção, o equipamento também contribui para o exame dos casos em face de sintomas compatíveis com a doença.

Além do uso de máscaras, que foram consideradas necessárias desde o início da pandemia, o desempenho na linha de frente da saúde também demonstrou efeitos sobre a prevalência de casos confirmados. ${ }^{27,28}$ Este resultado corrobora a prevalência da COVID-19 entre os enfermeiros registrados (estudantes de especialização, mestrado e doutorado e corpo docente), sendo maior do que os resultados gerais deste estudo. Um estudo prospectivo realizado nos EUA comparou os trabalhadores da linha de frente da saúde com a população geral dos EUA, demonstrando um maior risco de infecção entre os profissionais da saúde - $\mathrm{RH} 11,61$, IC 95\% 10,93-12,33. ${ }^{29}$ Uma situação semelhante foi identificada em um estudo transversal de soroconversão imunológica entre os prestadores de cuidados de saúde diretos em um ambiente de maternidade, demonstrando que a soroconversão estava provavelmente associada ao desempenho profissional e não necessariamente ao cenário de desempenho profissional. ${ }^{30}$

Seguindo a OMS e apesar de representar perdas na aquisição de habilidades práticas, a redução da exposição em ambientes de prática de cuidados deve ser considerada em planos futuros de reabertura de escolas, especialmente com estudantes de Enfermagem de nível inicial. ${ }^{31}$ Estes problemas indiscutíveis na educação em Enfermagem devem ser considerados pelas universidades e incluídos nos planos de recuperação de conteúdo curricular e aquisição de habilidades, que devem ocorrer pessoalmente após o fim da pandemia e após a vacinação em massa da população.

Além disso, o uso de protocolos entre as instituições educacionais para continuar as operações das aulas durante esta situação sem precedentes é uma necessidade (incentivar os princípios da tosse higiênica e espirros, o distanciamento físico, proporcionar instalações de lavagem e eliminação de resíduos, proporcionar um ambiente limpo e bem ventilado, fornecer máscaras para aqueles que não podem pagar, dar conselhos claros aos alunos sobre as ações que eles devem tomar se ficarem doentes ou pensarem que eles têm sintomas de COVID-19). As universidades devem ser aconselhadas a estabelecer uma resposta dedicada para aumentar o seu nível de preparação e resposta a fim de mitigar o impacto da COVID-19. A pandemia também traz a lição da necessidade de maiores investimentos em treinamento com a autoproteção dos estudantes e trabalhadores do setor de saúde.

A relação entre a infecção e o uso do transporte público também é um aspecto a ser considerado, especialmente quando se reconhecem as características dos grupos urbanos no Brasil e em outros países de baixa e média rendas. Destaca-se que uma parte significativa da comunidade acadêmica estudada utiliza o transporte público para deslocar-se de casa para a universidade. Assim, ao pensar na reabertura das Escolas de Enfermagem universitárias, isso também significa a necessidade de adotar um plano implementável de medidas de proteção para reduzir a exposição ao transporte público no caminho de ida e volta para a universidade. Os exemplos incluem as acomodações individuais ou em pequenos grupos e a implementação do monitoramento regular e sistemático dos sintomas e testes para a COVID-19 entre o corpo docente, o pessoal e os estudantes.

Uma constatação preocupante neste estudo foi o baixo nível de testes da COVID-19 na comunidade acadêmica estudada. Esta descoberta gerou um número significativo de casos não confirmados, mesmo entre os sujeitos que relataram as síndromes respiratórias. A maior prevalência de casos suspeitos está relacionada a subgrupos já listados na literatura tais como as minorias étnicas, as mulheres e os grupos de baixa renda. ${ }^{32-35}$ A pandemia acentuou as desigualdades na sociedade, que se tornaram mais visíveis e afetam estudantes, professores e técnicos administrativos nas universidades da mesma forma que, em geral, a população brasileira. ${ }^{36}$ 
Já é sabido que a falta de registros de infecção e de testes populacionais são grandes desafios para conter a pandemia, especialmente em regiões de baixa e média rendas. ${ }^{37} \mathrm{O}$ modelo matemático proposto pela Grassly e colegas (2020) reforça esta inferência ao mostrar o efeito dos testes semanais realizados por profissionais de saúde e do autoisolamento na presença de síndromes respiratórias como fatores protetores nas taxas de infecção pela COVID-19. Ao considerar a fase de estudos de ensaios clínicos sobre a vacina contra a SARS-CoV-2, qualquer plano para reabrir escolas e universidades, mesmo que gradualmente e com fluxo restrito de pessoas, deve contemplar a imunidade adquirida aparente e ainda contraditória após a infecção. ${ }^{38,39} \mathrm{~A}$ implementação da triagem em massa da COVID-19, incluindo o grupo de estudantes de saúde, é uma ação urgente e necessária.

Assim, os resultados da triagem da COVID-19 neste estudo, especialmente entre as categorias com menor exposição ao público (estudantes), tendem a produzir reflexões sobre a baixa porcentagem de imunidade à COVID-19 adquirida pela população. Com o possível e imediato retorno às aulas presenciais, podem ocorrer surtos da doença dentro das Escolas de Enfermagem, rompendo com a missão principal destes ambientes de treinamento. Além disso, parece importante contextualizar o alto risco de propagação da doença a partir dessas unidades acadêmicas, especialmente devido à maior exposição em estudantes que ainda estão em treinamento. Isso leva a um dilema: por um lado, enfermeiros que também são educadores têm a missão de cuidar e proteger; por outro lado, parece essencial impor a missão educacional para os futuros enfermeiros, mesmo em ambientes de risco. ${ }^{6}$

Apesar das importantes reflexões produzidas, os dados devem ser interpretados à luz de suas limitações. $O$ primeiro limite está centrado no desenho do estudo e na estratégia de amostragem. Como se tratou de uma pesquisa via web com amostragem de conveniência, a possibilidade de viés de seleção não deve ser descartada, especialmente quando se consideram os limites da conectividade digital no Brasil. A fim de contornar este problema de seleção, o estudo incluiu a fase de contatos telefônicos e acredita-se ter minimizado a seleção exclusiva de pessoas com acesso à Internet de boa qualidade. A opção de adotar os formulários Google como ferramenta para construir o questionário on-line também ajudou, aparentemente, a capacidade de resposta dos participantes. Por ser uma aplicação suave e comum utilizada no Brasil, acredita-se que este recurso também tenha ajudado as pessoas com dificuldades de acesso, permitindo que os participantes respondessem ao instrumento de pesquisa usando telefones celulares e tablets.

A segunda limitação estava na medição da prevalência da COVID-19, que se baseava no autorrelato da triagem da doença, assim como as datas da manifestação clínica, o contato com os casos positivos da doença, o tipo e o resultado dos testes diagnósticos. Assim, além do viés de memória, próprio dos itens de medida que exigiam retrospecção para as respostas, existe o problema de classificação resultante da multiplicidade de testes utilizados no Brasil. Nesses casos, há sempre a possibilidade de classificar melhor os casos positivos do que os negativos. Entretanto, com as respectivas ponderações resultantes das diferentes bases populacionais, é possível observar uma prevalência de $12,67 \%$ e $44,23 \%$, respectivamente, de resultados positivos em pacientes sintomáticos e negativos em pacientes assintomáticos testados. Apesar da variabilidade na precisão dos testes diagnósticos da COVID-19, a prevalência por resultados de subgrupos é compatível com as pesquisas sorológicas realizadas no Brasil e no mundo. Apesar de o modelo final não apresentar associações entre variáveis sociodemográficas e a prevalência da COVID-19, é essencial que novos estudos possam contemplar outras bases populacionais para um melhor exame desses efeitos.

\section{CONCLUSÃO}

O estudo mostrou uma prevalência de $11,65 \%$ de casos confirmados pela COVID-19, variando de acordo com a categoria acadêmica. O estudo identificou que $67,27 \%$ da amostra foi classificada como casos suspeitos e $21,08 \%$ como casos descartados. Entretanto, também foram observados testes baixos dos participantes do estudo. Apenas $24,79 \%$ da população investigada teve acesso a testes diagnósticos, mesmo aqueles que manifestaram síndromes respiratórias e incluindo as Síndromes Respiratórias Agudas Graves. O uso de transporte público e de máscaras e a participação na linha de frente da saúde foram fatores associados às diferenças entre os casos confirmados, descartados e suspeitos da COVID-19, possivelmente devido ao acesso aos testes da COVID-19.

A discussão dos planos de reabertura de escolas de Enfermagem no Brasil e no mundo deve integrar a discussão sobre o uso de equipamentos de proteção pessoal na educação, bem como nos ambientes de prática. As ações destinadas a aproximar os estudantes das escolas e universidades, reduzindo as viagens por transporte público, também são um tema de segurança a ser considerado. Finalmente, embora frequentemente realizada em muitos países do mundo, a triagem para a COVID-19, com maior acesso a testes laboratoriais, deve ser uma estratégia obrigatória para a reabertura de Escolas de Enfermagem.

\section{AGRADECIMENTOS}

As equipes da Escola de Enfermagem da Universidade do Estado do Rio de Janeiro (de trabalhadores e estudantes) desempenharam um papel fundamental durante a pandemia da COVID-19 e devem receber um sincero reconhecimento.

\section{FINANCIAMENTO}

Ricardo de Mattos Russo Rafael, Cristiane Helena Gallasch, Lucia Helena Garcia Penna e Helena Maria Scherlowski Leal David foram apoiados pelo financiamento do Programa de Incentivo à Produção Científica, Técnica e Artística da Universidade do Estado do Rio de Janeiro. Ricardo de Mattos Russo Rafael foi 
apoiado pelo Centro de Estudos e Pesquisas em Saúde Coletiva (CESPESC) da Universidade do Estado do Rio de Janeiro.

\section{CONTRIBUIÇÃO DOS AUTORES}

Concepção do desenho do estudo. Ricardo de Mattos Russo Rafael. Luiza Mara Correia. Alex Simões de Mello. Juliana Amaral Prata. Cristiane Helena Gallasch. Eugenio Fuentes Pérez Junior. Frances Valeria Costa e Silva. Lucia Helena Garcia Penna. Helena Maria Scherlowski Leal David.

Data de aquisição, Ricardo de Mattos Russo Rafael. Luiza Mara Correia. Alex Simões de Mello. Juliana Amaral Prata. Cristiane Helena Gallasch. Eugenio Fuentes Pérez Junior. Frances Valeria Costa e Silva. Lucia Helena Garcia Penna. Helena Maria Scherlowski Leal David.

Análise dos dados. Ricardo de Mattos Russo Rafael. Luiza Mara Correia. Alex Simões de Mello. Juliana Amaral Prata. Cristiane Helena Gallasch. Eugenio Fuentes Pérez Junior. Frances Valeria Costa e Silva. Lucia Helena Garcia Penna. Helena Maria Scherlowski Leal David.

Encontrar interpretação e discussão. Ricardo de Mattos Russo Rafael. Luiza Mara Correia. Alex Simões de Mello. Juliana Amaral Prata. Cristiane Helena Gallasch. Eugenio Fuentes Pérez Junior. Frances Valeria Costa e Silva. Lucia Helena Garcia Penna. Helena Maria Scherlowski Leal David. Jaime Caravaca Morera. Karen Lucas Breda.

Escrever e revisar criticamente o manuscrito. Ricardo de Mattos Russo Rafael. Luiza Mara Correia. Alex Simões de Mello. Juliana Amaral Prata. Cristiane Helena Gallasch. Eugenio Fuentes Pérez Junior. Frances Valeria Costa e Silva. Lucia Helena Garcia Penna. Helena Maria Scherlowski Leal David. Jaime Caravaca Morera. Karen Lucas Breda.

Aprovação final da versão do artigo. Ricardo de Mattos Russo Rafael. Luiza Mara Correia. Alex Simões de Mello. Juliana Amaral Prata. Cristiane Helena Gallasch. Eugenio Fuentes Pérez Junior. Frances Valeria Costa e Silva. Lucia Helena Garcia Penna. Helena Maria Scherlowski Leal David. Jaime Caravaca Morera. Karen Lucas Breda.

Assumir a responsabilidade de todos os aspectos do conteúdo do manuscrito, a exatidão e a integridade científica do artigo publicado. Ricardo de Mattos Russo Rafael. Luiza Mara Correia. Alex Simões de Mello. Juliana Amaral Prata. Cristiane Helena Gallasch. Eugenio Fuentes Pérez Junior. Frances Valeria Costa e Silva. Lucia Helena Garcia Penna. Helena Maria Scherlowski Leal David. Jaime Caravaca Morera. Karen Lucas Breda.

\section{EDITOR ASSOCIADO}

Cristina Rosa Baixinho

\section{REFERÊNCIAS}

1. World Health Organization. Rolling updates on coronavirus disease (COVID-19) [Internet]. Geneva:WHO; 2020 [cited 2020 Oct 2]. Available from: https://www.who.int/emergencies/diseases/novel-coronavirus-2019/ events-as-they-happen
2. World Health Organization. Naming the coronavirus disease (COVID-19) and the virus that causes it [Internet]. Geneva:WHO; 2020 [cited 2020 Oct 2]. Available from: https://www.who.int/emergencies/diseases/ novel-coronavirus-2019/technical-guidance/naming-the-coronavirusdisease-(covid-2019)-and-the-virus-that-causes-it

3. Rafael RMR, Neto M, de Carvalho MMB, David HMSL, Acioli S, Faria MGA. Epidemiology, public policies and covid-19 pandemics in Brazil: what can we expect? Rev Enferm. 2020;28:1-6. http://dx.doi.org/10.12957/ REUERJ.2020.49570.

4. Grassly NC, Pons-Salort M, Parker EPK, White PJ, Ferguson NM, Ainslie $K$ et al. Comparison of molecular testing strategies for COVID-19 control: a mathematical modelling study. Lancet Infect Dis. 2020;20(12):1381-1389. http://dx.doi.org/10.1016/S1473-3099(20)30630-7. PMid:32822577.

5. Yu HJ, HuYF, Liu X, Yao X, Wang Q, Liu L et al. Household infection: the predominant risk factor for close contacts of patients with COVID-19. Travel Med Infect Dis. 2020;36:101809. http://dx.doi.org/10.1016/j. tmaid.2020.101809. PMid:32592904.

6. McDonald CC. Reopening schools in the time of pandemic: look to the school nurses. J Sch Nurs. 2020;36(4):239-40. http://dx.doi. org/10.1177/1059840520937853. PMid:32552237.

7. Cooper DM, Guay-Woodford L, Blazar BR, Bowman S, Byington CL, Dome $\mathrm{J}$ et al. Reopening schools safely: the case for collaboration, constructive disruption of Pre-Coronavirus 2019 expectations, and creative solutions. J Pediatr. 2020;223:183-5. http://dx. doi.org/10.1016/j. jpeds.2020.05.022. PMid:32445649.

8. Li A, Harries M, Ross LF. Reopening K-12 Schools in the Era of Coronavirus Disease 2019: Review of State-Level Guidance Addressing Equity Concerns. J Pediatr. 2020;227:38-44.e7. http://dx.doi.org/10.1016/j. jpeds.2020.08.069. PMid:32866501.

9. Sheikh A, Sheikh A, Sheikh Z, Dhami S. Reopening schools after the COVID-19 lockdown. J Glob Health. 2020;10(1):1-3. http://dx.doi. org/10.7189/jogh.10.010376. PMid:32612815.

10. Viner RM, Bonell C, Drake L, Jourdan D, Davies N, Baltag V et al Reopening schools during the COVID-19 pandemic: governments must balance the uncertainty and risks of reopening schools against the clear harms associated with prolonged closure. Arch Dis Child. 2021 fev;106(2):111-3. http://dx.doi.org/10.1136/archdischild-2020-319963. PMid:32747375.

11. The Lancet.COVID-19 in Brazil: “So what?". Lancet. 2020;395(10235):1461 http://dx.doi.org/10.1016/S0140-6736(20)31095-3. PMid:32386576.

12. Mian A, Khan S. Coronavirus: the spread of misinformation. BMC Med. 2020;18(1):18-9. http://dx.doi.org/10.1186/s12916-020-01556-3. PMid:32188445.

13. Silva LV, Harb MPAA, Santos AMTB, Teixeira CAM, Gomes VHM, Cardoso EHS et al. COVID-19 mortality underreporting in Brazil: analysis of data from government internet portals. J Med Internet Res. 2020;22(8):1-14. http://dx.doi.org/10.2196/21413. PMid:32730219.

14. Von-Elm E, Altman DG, Egger M, Pocock SJ, Gøtzsche PC, Vandenbroucke $\mathrm{JP}$ et al. The strengthening the reporting of Observational Studies in Epidemiology (STROBE) statement: guidelines for reporting observational studies. PLoS Med. 2007;4(10):1623-7. http://dx.doi.org/10.1371/journal. pmed.0040296. PMid:17941714.

15. Eysenbach $\mathrm{G}$. Improving the quality of web surveys: the checklist for reporting results of internet E-Surveys (CHERRIES). J Med Internet Res. 2004;6(3):1-6. http://dx.doi.org/10.2196/jmir.6.3.e34. PMid:15471760.

16. Ministério da Saúde (BR). Protocolo de manejo clínico da Covid-19 na Atenção Especializada. Brasília: Ministério da Saúde; 2020.

17. StataCorp. Stata statistical software: release 15. Texas: StataCorp 2017.

18. Comar M, Brumat M, Concas MP, Argentini G, Bianco AR, Bicego L et al COVID-19 experience: first italian survey on healthcare staff members from a mother-child research hospital using combined molecular and rapid immunoassay tests. SSRN Electron J. 2020:1-12. https://doi. org/10.2139/ssrn.3592658.

19. Flannery D, Gouma S, Dhudasia M, Mukhopadhyay S, Pfeifer M, Woodford E, et al. SARS-CoV-2 seroprevalence among parturient women. medRxiv. No prelo 2020. https://doi.org/10.1101/2020.07.08. 20149179. 
20. Garcia-Basteiro AL, Moncunill G, Tortajada M, Vidal M, Guinovart C, Jiménez A et al. Seroprevalence of antibodies against SARS-CoV-2 among health care workers in a large Spanish reference hospital. Nat Commun. 2020;11(1):3500. http://dx.doi.org/10.1038/s41467-02017318-x. PMid:32641730.

21. Jerković I, Ljubić T, Bašić Ž, Kružić I, Kunac N, Bezić J et al. SARSCoV-2 antibody seroprevalence in industry workers in Split-Dalmatia and Šibenik-Knin County, Croatia. J Occup Environ Med. 2021 jan 1;63(1):32-7. http://dx.doi.org/10.1097/jom.0000000000002020. PMid:32925526.

22. Stringhini S, Wisniak A, Piumatti G, Azman AS, Lauer SA, Baysson $\mathrm{H}$ et al. Seroprevalence of anti-SARS-CoV-2 IgG antibodies in Geneva, Switzerland (SEROCoV-POP): a population-based study. Lancet. 2020;396(10247):313-9. http://dx.doi.org/10.1016/S0140-6736(20)313040. PMid:32534626.

23. Amorim L Fo, Szwarcwald CL, Mateos SOG, Ponce de Leon ACM, Medronho RA, Veloso VG et al. Seroprevalence of anti-SARS-CoV-2 among blood donors in Rio de Janeiro, Brazil. Rev Saude Publica. 2020;54:69. http://dx.doi.org/10.11606/s1518-8787.2020054002643. PMid:32638883.

24. Gomes CC, Cerutti C, Zandonade E, Maciel ELN, de Alencar FEC, Almada GL et al. A population-based study of the prevalence of COVID-19 infection in Espirito Santo, Brazil: methodology and results of the first stage. MedRxiv. 2020. http://dx.doi.org/10.1101/2020.06.1 3.20130559.

25. Hallal PC, Hartwig FP, Horta BL, Silveira MF, Struchiner CJ, Vidaletti LP et al. SARS-CoV-2 antibody prevalence in Brazil: results from two successive nationwide serological household surveys. Lancet Glob Heal. 2020;8(11):E1390-8. https://doi.org/10.1016/S2214-109X(20)30387-9.

26. Pollán M, Pérez-Gómez B, Pastor-Barriuso R, Oteo J, Hernán MA, Pérez-Olmeda $\mathrm{M}$ et al. Prevalence of SARS-CoV-2 in Spain (ENECOVID): a nationwide, population-based seroepidemiological study. Lancet. 2020;396(10250):535-44. http://dx.doi.org/10.1016/S01406736(20)31483-5. PMid:32645347.

27. Chu DK, Akl EA, Duda S, Solo K, Yaacoub S, Schünemann HJ et al. Physical distancing, face masks, and eye protection to prevent personto-person transmission of SARS-CoV-2 and COVID-19: a systematic review and meta-analysis. Lancet. 2020;395(10242):1973-87. http:// dx.doi.org/10.1016/S0140-6736(20)31142-9. PMid:32497510.

28. Wang C, Pan R, Tang S, Ji JS, Shi X. Mask use during COVID-19: a risk adjusted strategy. Environ Pollut. 2020 Nov;266(Pt 1):115099. http:// dx.doi.org/10.1016/j.envpol.2020.115099.

29. Nguyen LH, Drew DA, Graham MS, Joshi AD, Guo CG, Ma W et al. Risk of COVID-19 among front-line health-care workers and the general community: a prospective cohort study. Lancet Public Health. 2020;5(9):e475-83. http://dx.doi.org/10.1016/S2468-2667(20)30164-X. PMid:32745512.

30. Bampoe S, Lucas DN, Neall G, Sceales P, Aggarwal R, Caulfield K et al. A cross-sectional study of immune seroconversion to SARS-CoV-2 in frontline maternity health professionals. Anaesthesia. 2020;75(12):16149. http://dx.doi.org/10.1111/anae.15229. PMid:32777861.

31. World Health Organization. Considerations for school-related public health measures in the context of COVID-19: annex to considerations in adjusting public health and social measures in the context of COVID-19. Geneva:WHO; 2020.

32. Rafael RMR, Neto M, Depret DG, Gil AC, Fonseca MHS, SouzaSantos R. Effect of income on the cumulative incidence of COVID-19: an ecological study. Rev Lat Am Enfermagem. 2020;28:e3344. http:// dx.doi.org/10.1590/1518-8345.4475.3344. PMid:32609281.

33. Baqui P, Bica I, Marra V, Ercole A, van der Schaar M. Ethnic and regional variations in hospital mortality from COVID-19 in Brazil: a cross-sectional observational study. Lancet Glob Health. 2020;8(8):e1018-26. http:// dx.doi.org/10.1016/S2214-109X(20)30285-0. PMid:32622400.

34. Wenham C, Smith J, Morgan R, Gender and COVID-19 Working Group. COVID-19: the gendered impacts of the outbreak. Lancet. 2020;395(10227):846-8. http://dx.doi.org/10.1016/S0140-6736(20)305262. PMid:32151325.

35. Chattu VK, Yaya S. Emerging infectious diseases and outbreaks: Implications for women's reproductive health and rights in resourcepoor settings. Reprod Health. 2020;17(1):43. http://dx.doi.org/10.1186/ s12978-020-0899-y. PMid:32238177.

36. Minayo MCS, Freire NP. Pandemia exacerba desigualdades na Saúde. Cien Saude Colet. 2020;25(9):3555-6. http://dx.doi.org/10.1590/141381232020259.13742020. PMid:32876280.

37. Li R, Pei S, Chen B, Song Y, Zhang T, Yang W et al. Substantial undocumented infection facilitates the rapid dissemination of novel coronavirus (SARS-CoV-2). Science. 2020;368(6490):489-93. http:// dx.doi.org/10.1126/science.abb3221. PMid:32179701.

38. Manners C, Larios Bautista E, Sidoti H, Lopez OJ. Protective adaptive immunity against severe acute respiratory syndrome Coronaviruses 2 (SARS-CoV-2) and implications for vaccines. Cureus. 2020;12(6):e8399. http://dx.doi.org/10.7759/cureus.8399. PMid:32499988.

39. Ni L, Ye F, Cheng ML, Feng Y, Deng YQ, Zhao H et al. Detection of SARS-CoV-2-specific humoral and cellular immunity in COVID-19 convalescent individuals. Immunity. 2020;52(6):971-977.e3. http:// dx.doi.org/10.1016/j.immuni.2020.04.023. PMid:32413330. 